\title{
Erratum to: $\mathrm{Sn}^{4+}$ precursor enables $12.4 \%$ efficient kesterite solar cell from DMSO solution with open circuit voltage deficit below $0.30 \mathrm{~V}$ (vol 64, issue 1, page 52, 2021)
}

Yuancai Gong ${ }^{1}$, Yifan Zhang ${ }^{1}$, Erin Jedlicka ${ }^{2}$, Rajiv Giridharagopal ${ }^{2}$, James A. Clark ${ }^{3}$, Weibo Yan ${ }^{1}$, Chuanyou Niu ${ }^{1}$, Ruichan Qiu ${ }^{1}$, Jingjing Jiang, ${ }^{1}$, Shaotang $\mathrm{Yu}^{1}$, Sanping Wu ${ }^{1}$, Hugh W. Hillhouse ${ }^{3}$, David S. Ginger, Wei Huang and Hao Xin ${ }^{1 *}$

In the version of the article originally published in volume 64, issue 1, 2021 of Sci China Mater (2021, 64(1): 52-60, https://doi.org/10.1007/s40843-020-1408-x), the chemical formula of $\mathrm{Sn}(\mathrm{DMSO})_{4} \mathrm{Cl}_{4}$ (page $54,8^{\text {th }}$ line in the left column; page 56, $4^{\text {th }}$ and $6^{\text {th }}$ lines from the bottom of left column and in Equations (6 and 7) in the right column) was miswritten. The correct formula should be as below:

$\mathrm{SN}(\mathrm{DMSO})_{2} \mathrm{CL}_{4}$.

\footnotetext{
${ }^{1}$ Key Laboratory for Organic Electronics and Information Displays \& Jiangsu Key Laboratory for Biosensors, Institute of Advanced Materials (IAM), Jiangsu National Synergetic Innovation Center for Advanced Materials (SICAM), Nanjing University of Posts \& Telecommunications, Nanjing 210023, China

2 Department of Chemistry, University of Washington, Seattle, WA 98195 , USA

${ }^{3}$ Department of Chemical Engineering, University of Washington, Seattle, WA 98195, USA

* Corresponding author (email: iamhxin@njupt.edu.cn)
} 